haematopoetic malignancies and other cancers following occupational styrene exposure.

Method The cohort consists of 74902 workers (84\% men) in the Danish reinforced plastics industry, originating from 481 companies ever producing reinforced plastics in Denmark 19642009. We identified all workers in the National Supplementary Pension Fund Registry for which all employees are compulsory members. Cancer diagnoses were found in the National Cancer Registry. Standardised Incidence Rate Ratios (SIRs) and 95\% confidence intervals (95\% CI) were used for relative risk estimation. Results Among the 74902 workers, we identified 10374 cases of cancer accumulating 1.5 million person years. The overall SIR was 1.00 (95\% CI 0.98-1.02). SIR for lymphatic and haematopoietic cancers was 0.99 (0.91-1.07). Among male workers we observed increased risk of buccal cavity and pharygeal cancers (SIR 1.24; 1.12-1.37), cancers of the respiratory system (SIR $1.33 ; 1.26-1.39$ ), and bladder cancer (SIR $1.08 ; 1.0-1.17$ ), and among female workers cancers of the respiratory system (SIR $1.41 ; 1.22-1.62)$.

Conclusions The cohort experiences the same overall cancer risk as the general population and no increased overall risk of malignant haematopoietic diseases was apparent. However, we observed increased risks for cancers that may be due to confounding from smoking and alcohol. Internal risk assessment that includes historical styrene exposure data will supplement the current findings.

\section{QUALITY OF LIFE OF WORKERS SUFFERING FROM SHOULDER PAIN}

${ }^{1}$ Julie Bodin, ${ }^{2,3}$ Ronan Garlantézec, ${ }^{4}$ Alexis Descatha, ${ }^{5}$ Catherine $\mathrm{Ha},{ }^{1,6}$ Yves Roquelaure. 'LUNAM University, University of Angers, Laboratory of Ergonomics and Epidemiology in Occupational Health (LEEST), Angers, France; 2IRSET INSERM U1085, Univ Rennes I, Rennes, France; ${ }^{3}$ EHESP, School of Public Heath, Rennes, France; ${ }^{4}$ nserm, Centre for Research in Epidemiology and Population Health (CESP), U1018, "Population-Based Epidemiological Cohorts" Research Platform, F-94807, Villejuif, France; ${ }^{5}$ French Institute for Public Health Surveillance, Department of Occupational Health, Saint-Maurice, France; ${ }^{6} \mathrm{CHU}$ Angers, Angers, France

\subsection{6/oemed-2014-102362.258}

Objectives To compare the quality of life (QoL) in three groups of workers suffering or not from shoulder pain (SP) lasting more than one month during the preceding 12 months.

Method Between 2002-2005, 3710 workers were randomly included in a French surveillance system of work-related musculoskeletal disorders. In 2007, 2332 responded to a follow-up questionnaire, 2049 were still active. Workers completed the Nordic Questionnaire to assess SP and the SF-36 for QoL. Three groups were defined according to health status at follow-up:

- Group 1: workers without SP (men: 87.9\%; women: 79.2\%)

- Group 2: workers with SP without neck, elbow and hand/ wrist pain lasting more than one month during the preceding 12 months (men: 4.2\%; women: 6.0\%)

- Group 3: workers with SP and neck, elbow or hand/wrist pain lasting more than one month during the preceding 12 months (men 7.9\%; women 14.8\%)

The mean scores of SF-36 were compared with Kruskall-Wallis test and post-hoc comparisons were performed. Analyses were stratified by gender.

Results Workers in group 2 had lower scores of physical health compared to workers in group 1, whatever the gender. Workers in group 3 had lower scores of physical and mental health compared to workers in group 1 . Two dimensions of mental health in men and the four dimensions of physical health and one dimension of mental health in women had lower scores in group 3 compared to group 2 .

Conclusions Workers with SP and upper-limb pain have poorer QoL compared to workers without SP and workers with SP without upper-limb pain.

\section{INDEPENDENT MEDICAL EVALUATIONS - IMPORTANT, NEGLECTED, IN NEED OF REFORM: A SYSTEMATIC REVIEW}

${ }^{1}$ Jason Busse, 'Shanil Ebrahim, 'John Riva, ${ }^{2}$ Sheena Bance, 'Gordon Guyatt, ${ }^{2}$ Michael Bagby, ${ }^{3}$ Regina Kunz. ${ }^{1}$ McMaster University, Hamilton, Ontario, Canada; ${ }^{2}$ University of Toronto, Toronto, Ontario, Canada; ${ }^{3}$ Basel Academy of Swiss Insurance Medicine, Basel, Switzerland

\subsection{6/oemed-2014-102362.259}

Objectives Independent medical evaluations (IMEs) are a common and influential form of assessment, often influencing whether patients receive compensation for an injury or illness. To inform the evidence-base underlying IMEs, we conducted a systematic review of all primary literature conducted in North America.

Method We searched CINAHL, EMBASE, MEDLINE and PsycINFO and other sources for studies published through to Sept. 20, 2011. We included all primary literature on the topic of IMEs from a North American perspective. Assessment for study inclusion, data extraction and risk-of-bias analyses were performed in duplicate.

Results We included 52 studies, all of which were observational in design and most of which focussed on determining the rate of malingering among examinees. Estimates of non-credible symptom over-reporting among patients presenting for IMEs ranged from $16 \%$ to $55 \%$, with studies at lower risk of bias finding higher estimates. Other studies found that inter-rater reliability among IME assessors for assigning degree of impairment to the same IME report was poor, and that patients presenting for an IME with external incentive (e.g. litigation, disability benefits) perform systematically worse across a range of psychometric tests versus patients presenting with similar illness/injury but without external incentive.

Conclusions Symptom exaggeration is common among patients presenting for IMEs, and particularly among those patients with external incentive. IME assessors reviewing the same case demonstrate little agreement regarding the degree of impairment that should be assigned. Standards for IME assessment and reporting are urgently needed to ensure greater reliability and validity of this common form of assessment.

\section{DOES LONG-TERM STRESS CAUSE DEPRESSION? OCCUPATIONAL NOISE EXPOSURE AND THE USE OF ANTIDEPRESSANTS}

${ }^{1} \mathrm{NM}$ Raunkjaer, ${ }^{2}$ ZA Stokholm, ${ }^{3} \mathrm{MV}$ Willert, ${ }^{4} \mathrm{O}$ Mors, ${ }^{5} \mathrm{JM}$ Vestergaard, ${ }^{6} \mathrm{TW}$ Frederiksen, 7 HA Kolstad. 'Danish Ramazzini Centre, Department of Occupational Medicine, Aarhus University Hospital, Aarhus, Denmark; ${ }^{2}$ Danish Ramazzini Centre, Department of Occupational Medicine, Aarhus University Hospital, Aarhus, Denmark; ${ }^{3}$ Danish Ramazzini Centre, Department of Occupational Medicine, Aarhus University Hospital, Aarhus, Denmark; ${ }^{4}$ Research Department P, Risskov, Denmark; ${ }^{5}$ Danish Ramazzini Centre, Department of Occupational Medicine, Aarhus University Hospital, Aarhus, Denmark; ${ }^{6}$ Danish Ramazzini Centre, Department of Occupational Medicine, Aarhus University Hospital, Aarhus, Denmark; ${ }^{7}$ Danish Ramazzini Centre, Department of Occupational Medicine, Aarhus University Hospital, Aarhus, Denmark

10.1136/oemed-2014-102362.260 